\title{
Bruhat-Chevalley Order in Reductive Monoids
}

\author{
MOHAN S. PUTCHA \\ Department of Mathematics, Box 8205, North Carolina State University, Raleigh, NC 27695-8205
}

Received January 24, 2003; Revised July 7, 2003; Accepted August 5, 2003

\begin{abstract}
Let $M$ be a reductive monoid with unit group $G$. Let $\Lambda$ denote the idempotent cross-section of the $G \times G$-orbits on $M$. If $W$ is the Weyl group of $G$ and $e, f \in \Lambda$ with $e \leq f$, we introduce a projection map from $W e W$ to $W f W$. We use these projection maps to obtain a new description of the Bruhat-Chevalley order on the Renner monoid of $M$. For the canonical compactification $X$ of a semisimple group $G_{0}$ with Borel subgroup $B_{0}$ of $G_{0}$, we show that the poset of $B_{0} \times B_{0}$-orbits of $X$ (with respect to Zariski closure inclusion) is Eulerian.
\end{abstract}

Keywords: reductive monoid, Renner monoid, Bruhat-Chevalley order, projections

2000 Mathematics Subject Classification: Primary 20G99, 20M99, 06A07

\section{Introduction}

Reductive monoids $M$ are Zariski closures of reductive groups $G$. By this we mean that if $G$ is a closed subgroup of $G L_{n}(k)$, then the closure $M$ of $G$ in $M_{n}(k)$ is a reductive monoid. The Bruhat-Chevalley order in $G$ has a natural extension to reductive monoids. The Renner monoid $R$ takes the place of the Weyl group $W$. Associated with the Bruhat decomposition of a $G \times G$-orbit of $M$ is a $W \times W$-orbit of $R$ that is a graded poset and has been explicitly determined by the author [7]. The ordering on $R$ is more removed from the ordering on $W$ and hence harder to understand. It is the detailed study of the ordering on $R$ that is the purpose of this paper.

The $W \times W$-orbits are indexed by the cross-section lattice $\Lambda$ of $M$. For two $W \times W$-orbits $W e W, W f W$ with $e \leq f$, we define an upward projection map $p: W e W \rightarrow W f W$. These are order-preserving maps with some pleasing properties. If $\sigma \in W e W, \theta \in W f W$, then we show that $\sigma \leq \theta$ in $R$ if and only if $p(\sigma) \leq \theta$. Combining with the description of the order on the $W \times W$-orbits in [7], we obtain a new description of the order on $R$ which enables us to obtain several consequences. In particular we show that any length 2 interval in $R$ is either a chain or a diamond. This leads to a conjecture on the Möbius function on $R$.

We go on to study canonical reductive monoids associated with the canonical compactification of semisimple groups, cf. [13]. We prove that for a canonical monoid, the poset $R^{*}=R \backslash\{0\}$ is Eulerian. This extends a classical result of Verma [18] that $W$ is Eulerian and a recent result of the author [7] that the $W \times W$-orbits in $R^{*}$ are Eulerian. 


\section{Preliminaries}

Let $P$ be a finite partially ordered set with a maximum and minimum element such that all maximal chains have the same length. Then $P$ admits a rank function with the minimal element having rank zero. If $X \subseteq P$, we will say that $X$ is balanced if the number of even rank elements of $X$ is equal to the number of odd rank elements of $X$. $P$ is said to be Eulerian if for $\alpha, \beta \in P$ with $\alpha<\beta$, the interval $[\alpha, \beta]$ is balanced. Eulerian posets were first defined by Stanley [16] and have been extensively studied. The Dehn-Somerville equations are valid in these posets and as stated in [17; Section 3.14], Eulerian posets enjoy remarkable duality properties.

Let $k$ be an algebraically closed field and let $G$ be a reductive group defined over $k$. Let $T$ be a maximal torus contained in a Borel subgroup $B$ of $G$. Let $W=N_{G}(T) / T$ denote the Weyl group of $G$ and let $S$ denote the generating set of simple reflections of $W$. Then $G$ has the Bruhat decomposition:

$$
G=\bigsqcup_{w \in W} B w B
$$

As in [1], the Bruhat-Chevalley order on $W$ is defined as:

$$
x \leq y \quad \text { if } B x B \subseteq \overline{B y B}
$$

As is well known, this is equivalent $x$ being a subword of a reduced expression $y=$ $\alpha_{1} \ldots \alpha_{m}, \alpha_{1}, \ldots, \alpha_{m} \in S$. The length $l(y)$ is defined to be $m$. If $w_{0}$ is the longest element of $W$, then $B^{-}=w_{0} B w_{0}$ is the Borel subgroup of $G$ opposite to $B$ relative to $T$. If $x_{1}, \ldots, x_{n} \in W$, then let

$$
x_{1} * \cdots * x_{n}= \begin{cases}x_{1} \ldots x_{n} & \text { if } l\left(x_{1} \ldots x_{n}\right)=l\left(x_{1}\right)+\cdots+l\left(x_{n}\right) \\ \text { undefined } & \text { otherwise }\end{cases}
$$

For $x, y \in W$, let $x \circ y, x \Delta y \in W$ be defined as:

$$
\overline{B(x \circ y) B}=\overline{B x B y B}, \overline{B^{-}(x \triangle y) B}=\overline{B^{-} x B y B}
$$

Lemma 1.1 Let $x, y \in W$. Then

(i) $x \circ y=x_{1} * y=x * y_{1}$ for some $x_{1} \leq x, y_{1} \leq y$

(ii) $x \circ y=\max \left\{x y^{\prime} \mid y^{\prime} \leq y\right\}=\max \left\{x^{\prime} y \mid x^{\prime} \leq x\right\}=\max \left\{x^{\prime} y^{\prime} \mid x^{\prime} \leq x, y^{\prime} \leq y\right\}$

(iii) $x \Delta y=\min \left\{x y^{\prime} \mid y^{\prime} \leq y\right\}=\min \left\{x^{\prime} y \mid x^{\prime} \geq x\right\}=\min \left\{x^{\prime} y^{\prime} \mid x^{\prime} \geq x, y^{\prime} \leq y\right\}$

(iv) $x \Delta y=x y_{1}$ with $y_{1} \leq y$ and $x=\left(x y_{1}\right) * y_{1}^{-1}$

(v) $x \Delta y=x y$ if and only if $l(x y)=l(x)-l(y)$

(vi) $(x \Delta y) \circ y^{-1}=(x \Delta y) y^{-1}$ and $(x \circ y) \Delta y^{-1}=(x \circ y) y^{-1}$

Proof: (i) follows the Tits axioms and induction on length. (ii) then follows from (i) and (3). (iii) and (iv) follow from (i) and (ii) by noting that $x \Delta y=w_{0}\left(\left(w_{0} x\right) \circ y\right)$. 
(iv) Let $x \Delta y=s$. Then by (iv) $x=s y_{1}^{-1}$ for some $y_{1} \leq y$. Then by (i), (ii),

$$
x=s y_{1}^{-1} \leq s \circ y_{1}^{-1} \leq s \circ y^{-1}=s^{\prime} * y^{-1}
$$

for some $s^{\prime} \leq s$. So $x=s^{\prime \prime} * y_{2}^{-1}$ for some $s^{\prime \prime} \leq s^{\prime}, y_{2} \leq y$. So

$$
s \geq s^{\prime} \geq s^{\prime \prime}=x y_{2}^{-1} \geq x \Delta y=s
$$

Hence $s=s^{\prime}$ and $(x \Delta y) \circ y^{-1}=(x \Delta y) y^{-1}$.

Corollary 1.2 Let $x, x^{\prime}, y, y^{\prime} \in W$. If $x \geq x^{\prime}$ and $x * y=x^{\prime} * y^{\prime}$, then $y \leq y^{\prime}$.

Proof: By Lemma 1.1,

$$
x * y=x^{\prime} * y^{\prime}=x^{\prime} \circ y^{\prime} \leq x \circ y^{\prime}=x * y_{1}
$$

for some $y_{1} \leq y^{\prime}$. By [7; Lemma 2.1], $y \leq y_{1}$. So $y \leq y^{\prime}$.

Lemma 1.3 Let $x_{0}, y_{0}, s \in W$ such that $s_{0}=x_{0} \Delta y_{0} \leq s$. Then

$$
Y=\left\{y \leq y_{0}^{-1} \mid s \circ y=x_{0}\right\}
$$

is a balanced subset of $W$.

Proof: By Lemma 1.1 (iv), $s_{0}=x_{0} y_{1}$, with $y_{1} \leq y_{0}$ and $x_{0}=s_{0} * y_{1}^{-1}$. Let $y \in Y$. Then $y \leq y_{0}^{-1}$ and $s \circ y=x_{0}$. By Lemma 1.1 (i), $x_{0}=s^{\prime} * y$ with $s^{\prime} \leq s$. Then

$$
s_{0}=x_{0} \Delta y_{0} \leq x_{0} y^{-1}=s^{\prime}
$$

Since $s_{0} * y_{1}^{-1}=x_{0}=s^{\prime} * y$, we see by Corollary 1.2 that $y \leq y_{1}^{-1}$. Hence

$$
Y=\left\{y \in W \mid y \leq y_{1}^{-1}, x_{0}=s \circ y\right\}
$$

We prove by induction on $l\left(y_{1}\right)$ that $Y$ is balanced. If $l\left(y_{1}\right)=0$, then $x_{0}=s_{0}<s$ and $Y=\emptyset$. So let $l\left(y_{1}\right)>0$. Let $y_{1}=\alpha * y_{2}, \alpha \in S$. Let

$$
\begin{aligned}
& Y_{1}=\{y \in Y \mid y \alpha>y\} \\
& Y_{2}=\{y \in Y \mid y \alpha<y, y \alpha \in Y\}
\end{aligned}
$$

Let $y \in Y_{1}$. Then $y \leq y_{1}^{-1}$. So

$$
y \alpha=y \circ \alpha \leq y_{1}^{-1} \circ \alpha=y_{1}^{-1}
$$


and

$$
s \circ(y \alpha)=s \circ y \circ \alpha=x_{0} \circ \alpha=s_{0} \circ y_{1}^{-1} \circ \alpha=s_{0} \circ y_{1}^{-1}=x_{0}
$$

Hence $y \alpha \in Y_{2}$. Thus $Y_{1} \sqcup Y_{2}$ is balanced. Let

$$
Y_{3}=\{y \in Y \mid y \alpha<y, y \alpha \notin Y\}
$$

So $Y=Y_{1} \sqcup Y_{2} \sqcup Y_{3}$. Let $x_{1}=s_{0} y_{2}^{-1}=s_{0} * y_{2}^{-1}$. Then by induction hypothesis,

$$
Z=\left\{y \in Y \mid y \leq y_{2}^{-1}, s \circ y=x_{1}\right\}
$$

is balanced. Let $y \in Z$. Then $x_{1}=s \circ y=s_{1} * y$ for some $s_{1} \leq s$. Since $x_{1}<x_{1} \alpha$, we see that $y<y \alpha$. Then

$$
y \alpha=y \circ \alpha \leq y_{2}^{-1} \circ \alpha=y_{1}^{-1}
$$

and

$$
s \circ(y \alpha)=s \circ y \circ \alpha=x_{1} \circ \alpha=x_{0}
$$

Thus $y \alpha \in Y_{3}$. Conversely let $y \in Y_{3}$. Then

$$
(y \alpha) * \alpha=y \leq y_{1}^{-1}=y_{2}^{-1} * \alpha
$$

Hence $y \alpha \leq y_{2}^{-1}$. Also $s \circ(y \alpha) \neq x_{0}$ and

$$
(s \circ(y \alpha)) \circ \alpha=s \circ((y \alpha) \circ \alpha)=s \circ y=x_{0}
$$

Thus

$$
(s \circ(y \alpha)) * \alpha=x_{0}=x_{1} * \alpha
$$

So $s \circ(y \alpha)=x_{1}$ and $y \alpha \in Z$. Since $Z$ is balanced, we see that $Y_{3}$ is balanced. Hence $Y=Y_{1} \sqcup Y_{2} \sqcup Y_{3}$ is balanced.

If $I \subseteq S$, then as usual let $W_{I}$ denote the parabolic subgroup of $W$ generated by $I$ and let

$$
D_{I}=\left\{x \in W \mid x w=x * w \quad \text { for all } w \in W_{I}\right\}
$$

denote the set of minimal length left coset representatives of $W_{I}$. 
Let $M$ be a reductive monoid having $G$ as its unit group. Thus $M$ is the Zariski closure of $G$ in some $M_{n}(k)$, where $M_{n}(k)$ is the monoid of all $n \times n$ matrices over $k$. We refer to $[6,14]$ for details. The idempotent set $E(\bar{T})$ of $\bar{T}$ is a finite lattice isomorphic to the face lattice of a rational polytope. As in [5], let

$$
\Lambda=\{e \in E(\bar{T}) \mid B e=e B e\}
$$

Then $\Lambda$ is a cross-section of the $G \times G$-orbits of $M$ such that for all $e, f \in \Lambda$,

$$
e \leq f \Leftrightarrow e \in M f M
$$

Here as usual, $e \leq f$ means $e f=e=f e . \Lambda$ is called the cross-section lattice of $M$. All maximal chains in $\Lambda$ have the same length. We note that for $M_{n}(k)$,

$$
\Lambda=\left\{\left[\begin{array}{ll}
I_{r} & 0 \\
0 & 0
\end{array}\right] \mid 0 \leq r \leq n\right\}
$$

is the usual set of idempotent representatives of matrices of different ranks.

By [10], the Bruhat decomposition (1) is extended to $M$ as

$$
M=\bigsqcup_{\sigma \in R} B \sigma B
$$

where $R=\overline{N_{G}(T)} / T$ is the Renner monoid of $M . W$ is the unit group of $R$ and

$$
R=\bigsqcup_{e \in \Lambda} W e W
$$

The Bruhat-Chevalley order (2) on $W$ extends to $R$ as:

$$
\sigma \leq \theta \quad \text { if } B \sigma B \subseteq \overline{B \theta B}
$$

Then each WeW is an interval in $R$ and by [10] all maximal chains in $R$ have the same length. $R$ is an inverse semigroup. This means that the map, $\sigma \rightarrow \sigma^{-1}$ is an involution of $R$. Here if $\sigma=x e y \in W e W$, then $\sigma^{-1}=y^{-1} e x^{-1}$. Unlike in $W$, this involution is not order preserving. However by [11], the map

$$
\sigma \rightarrow w_{0} \sigma^{-1} w_{0}
$$

is an order preserving involution of $R$. Let $e \in \Lambda$. Then as in [8], consider (in $R$ ),

$$
\lambda(e)=\{s \in S \mid s e=e s\}
$$


and

$$
\lambda^{*}(e)=\bigcap_{f \geq e} \lambda(f), \quad \lambda_{*}(e)=\bigcap_{f \leq e} \lambda(f)
$$

Then

$$
\begin{aligned}
W(e) & =W_{\lambda(e)}=\{w \in W \mid w e=e w\}, \\
W^{*}(e) & =W_{\lambda^{*}(e)}, \\
W_{*}(e) & =W_{\lambda_{*}(e)}=\{w \in W \mid w e=e=e w\}
\end{aligned}
$$

are parabolic subgroups of $W$ with

$$
W(e)=W^{*}(e) \times W_{*}(e)
$$

Moreover $W^{*}(e)$ is the Weyl group of the unit group of $e$ Me. See [6; Chapter 10] for details. If $I=\lambda(e), K=\lambda_{*}(e)$, let

$$
D(e)=D_{I}, \quad D_{*}(e)=D_{K}
$$

Let

$$
\mathcal{W}_{I, K}^{*}=D_{I} \times W_{I \backslash K} \times D_{I}^{-1}
$$

For $\sigma=(x, w, y), \sigma^{\prime}=\left(x^{\prime}, w^{\prime}, y^{\prime}\right) \in \mathcal{W}_{I, K}^{*}$, define

$$
\sigma \leq \sigma^{\prime} \quad \text { if } w=w_{1} * w_{2} * w_{3} \quad \text { with } \quad x w_{1} \leq x^{\prime}, w_{2} \leq w^{\prime}, w_{3} y \leq y^{\prime}
$$

By [7; Theorem 2.5],

$$
\mathcal{W}_{I, K}^{*} \text { is isomorphic to the dual of WeW }
$$

The order on $R$ is more subtle. Let $\sigma \in R$. Then

$$
\sigma=x e y \quad \text { for unique } e \in \Lambda, x \in D_{*}(e), y \in D(e)^{-1}
$$

This is called the standard form of $\sigma$. Let $\sigma=x e y, \theta=s f t \in R$ in standard form. Then by [4],

$$
\sigma \leq \theta \Leftrightarrow e \leq f, \quad x \leq s w, \quad w^{-1} t \leq y \quad \text { for some } w \in W(f) W_{*}(e)
$$


Let $\sigma=x e y$ be in standard form. Let $x \leq x_{1}, y_{1} \leq y$. Let $y_{1}=u y_{2}, u \in W(e), y_{2} \in$ $D(e)^{-1}$. Let $x_{1} u=x_{2} z, x_{2} \in D_{*}(e), z \in W_{*}(e)$. Then $x_{1} e y_{1}=x_{2} e y_{2}$ in standard form. Now

$$
x \leq x_{1}=x_{2} z u^{-1}=x_{2} u^{-1} \cdot u z u^{-1}
$$

Since $u z u^{-1} \in W_{*}(e)$ and $x \in D_{*}(e), x \leq x_{2} u^{-1}$. Also $u y_{2}=y_{1} \leq y$. Hence $\sigma \leq x_{2} e y_{2}$. Thus,

$$
\sigma=x e y \text { in standard form } \Rightarrow \sigma \leq x_{1} e y_{1} \text { for all } x_{1} \geq x, y_{1} \leq y
$$

If $e, f \in \Lambda$ with $e \leq f$, then $e \in \overline{f T}$ and so we see directly from (6) that

$$
x e y \leq x f y \quad \text { for all } x, y \in W
$$

The length function on $R$ is defined as follows. Let $\sigma=x e y$ in standard form. Then

$$
l(\sigma)=l(x)+l(e)-l(y)
$$

where $l(e)$ is the length of the longest element in $D(e)$. We refer to $[4,7,11,14]$ for further details. In particular,

$$
\text { length function }=\text { rank function on } W e W, e \in \Lambda
$$

where the rank function is determined from the grading of $W e W$.

\section{Projections}

We wish to better understand the order $\leq$ on $\mathrm{R}$ given by (6), (16). For $e \in \Lambda$, let $z_{\mathrm{e}}$ denote the longest element in $W_{*}(e)$. Let $e, f \in \Lambda$ with $e \leq f$. Let $\sigma=x e y \in W e W$ in standard form. Let $z_{e} y=u y_{1}, u \in W(f), y_{1} \in D(f)^{-1}$. We define the projection of $\sigma$ in $W f W$ as:

$$
p_{e, f}(\sigma)=(x \Delta u) f y_{1}
$$

We claim that (21) is in standard form. Let $x=x_{1} v, x_{1} \in D(f), v \in W(f)$. Since $x \in$ $D_{*}(e)$ and $W_{*}(f) \subseteq W_{*}(e)$, we see that $v \in W^{*}(f)$. By (10), $v \Delta u \in W^{*}(f)$. Thus $x \Delta u=$ $x_{1}(v \Delta u) \in D_{*}(f)$. Hence (21) is in standard form. Now $z_{f} z_{e} y=u^{\prime} y_{1}$ with $u^{\prime}=z_{f} u \in$ $W(f)$ and by the above, $v \Delta u^{\prime}=v \Delta u$. Hence we also have

$$
p_{e, f}(\sigma)=\left(x \Delta u^{\prime}\right) f y_{1} \text { in standard form }
$$

The following result in conjunction with (14) yields a new description of the order on $R$. 
Theorem 2.1 Let $e, f \in \Lambda, e \leq f$. Then

(i) $p_{e, f}: W e W \rightarrow W f W$ is order preserving and $\sigma \leq p_{e, f}(\sigma)$ for all $\sigma \in W e W$.

(ii) If $\sigma \in W e W, \theta \in W f W$, then $\sigma \leq \theta$ if and only if $p_{e, f}(\sigma) \leq \theta$.

(iii) If $h \in \Lambda$ with $e \leq h \leq f$, then $p_{e, f}=p_{h, f} \circ p_{e, h}$.

(iv) $p_{e, f}$ is onto if and only if $\lambda_{*}(e) \subseteq \lambda_{*}(f)$.

(v) $p_{e, f}$ is $1-1$ if and only if $\lambda(f) \subseteq \lambda(e)$.

Proof: Let $\sigma=x e y$ in standard form. Let $z_{e} y=u y_{1}, u \in W(f), y_{1} \in D(f)^{-1}$. By Lemma 1.1, $x \Delta u=x u_{1}$ with $u_{1} \leq u$. Then

$$
u_{1} y_{1} \leq u y_{1}=z_{e} y
$$

Hence $u_{1} y_{1}=z y^{\prime}$ for some $z \leq z_{1}, y^{\prime} \leq y$. Then $z \in W_{*}(e)$ and $\left(z^{-1} u_{1}\right) y_{1}=y^{\prime} \leq y$. Also since $x \in D_{*}(e)$,

$$
x \leq x z=\left(x u_{1}\right)\left(u_{1}^{-1} z\right)
$$

By (16), (21),

$$
\sigma=x e y \leq x u_{1} f y_{1}=p_{e, f}(\sigma)
$$

Let $\theta=s f t$ in standard form such that $\sigma \leq \theta$. Then by (16),

$$
x \leq s w, w^{-1} t \leq y \quad \text { for some } w \in W(f) W_{*}(e)
$$

So $x=s_{1} * w_{1}$ for some $s_{1} \leq s, w_{1} \leq w$. Since $x \in D_{*}(e), w_{1} \in D_{*}(e)$. Now $w=w_{2} * z$ for some $w_{2} \in W(f), z \in W_{*}(e)$. Then $w_{1} \leq w_{2}$. Since $t \in D(f)^{-1}$ and $y \in D(e)^{-1}$,

$$
w_{1}^{-1} t \leq w_{2}^{-1} t=z z^{-1} w_{2}^{-1} t=z w^{-1} t \leq z \circ\left(w^{-1} t\right) \leq z \circ y \leq z_{e} \circ y=z_{e} y=u y_{1}
$$

Since $t, y_{1} \in D(f)^{-1}$ and $w_{1}, u \in W(f)$, we see by [7; Lemma 2.2] that $w_{1}=w_{3} * w_{4}$ with $w_{4}^{-1} \leq u, w_{3}^{-1} t \leq y_{1}$. So

$$
x \Delta u \leq x w_{4}^{-1}=s_{1} w_{1} w_{4}^{-1}=s_{1} w_{3} \leq s \circ w_{3}=s * w_{5}
$$

for some $w_{5} \leq w_{3}$. Also $w_{5}^{-1} t \leq w_{3}^{-1} t \leq y_{1}$. Hence by (21),

$$
p_{e, f}(\sigma)=(x \Delta u) f y_{1} \leq s f t=\theta
$$

So if $\sigma^{\prime} \in W e W$ with $\sigma \leq \sigma^{\prime}$, then by (23), $\sigma \leq \sigma^{\prime} \leq p_{e, f}\left(\sigma^{\prime}\right)$. So by (24), $p_{e, f}(\sigma) \leq$ $p_{e, f}\left(\sigma^{\prime}\right)$. This proves (i), (ii). 
Let $e \leq h \leq f$ in $\Lambda$. Let $\sigma \in W e W, \theta=p_{e, f}(\sigma)$. Then by (i), (ii), $p_{e, f}(\sigma) \leq p_{h, f} \circ$ $p_{e, h}(\sigma)$. Let $\sigma=x e y, \theta=s f t$ in standard form. Then by (16), $x \leq s w, w^{-1} t \leq y$ for some $w \in W(f) W_{*}(e)$. So $w=w_{1} * z$ for some $w_{1} \in W(f), z \in W_{*}(e)$. Then by (17), (18),

$$
\sigma=x e y \leq s w e w^{-1} t=s w_{1} e w_{1}^{-1} t \leq s w_{1} h w_{1}^{-1} t \leq s w_{1} f w_{1}^{-1} t=\theta
$$

So if $\pi=s w_{1} h w_{1}^{-1} t \in W h W$, then $\sigma \leq \pi \leq \theta$. By (ii), $p_{e, f}(\sigma) \leq \pi$ and $p_{h, f}(\pi) \leq \theta$. So by (i),

$$
p_{h, f} \circ p_{e, f}(\sigma) \leq p_{h, f}(\pi) \leq \theta=p_{e, f}(\sigma)
$$

This proves (iii).

(iv) Suppose first that $\lambda_{*}(e) \subseteq \lambda_{*}(f)$. By (9), $\lambda^{*}(e) \subseteq \lambda^{*}(f)$. So $\lambda(e) \subseteq \lambda(f)$. Hence $W(e) \subseteq W(f)$ and $W_{*}(e)=W_{*}(f)$. So $D_{*}(e)=D_{*}(f)$ and $D(f) \subseteq D(e)$. Thus if $\theta=x f y \in W f W$ is in standard form, then $\sigma=x e y$ is in standard form and $p_{e, f}(\sigma)=\theta$. Thus $p_{e, f}$ is onto.

Assume conversely that $p_{e, f}$ is onto. Let $w$ be the longest element in $W^{*}(f)$ and let $\theta=w f \in W f W$. There exists $\sigma=x e y$ in standard form such that $p_{e, f}(\sigma)=\theta$. By (21), $w=x \Delta u$ for some $u \in W(f)$. So $w \leq x$ and $x \in W(f)$. By (9), (11), $x \in D_{*}(e) \subseteq D_{*}(f)$. So by (10), $x \in W^{*}(f)$. Thus $x=w$. Since $w$ is the longest element of $W^{*}(f) x, \alpha<x$ for all $\alpha \in \lambda^{*}(f)$. Since $x \in D_{*}(e), x \alpha>x$ for all $\alpha \in \lambda_{*}(e)$. Hence $\lambda^{*}(e) \cap \lambda_{*}(f)=\emptyset$. There exists $x_{1} e y_{1}$ in standard form such that $p_{e, f}\left(x_{1} e y_{1}\right)=f$. By $(21), z_{e} * y_{1} \in W(f)$. Hence by $(10), z_{e} \in W(f)=W^{*}(f) \times W_{*}(f)$. Since $W_{*}(e) \cap W^{*}(f)=\{1\}, z_{e} \in W_{*}(f)$. Hence $\lambda_{*}(e) \subseteq \lambda_{*}(f)$.

(v) Let $\lambda(f) \subseteq \lambda(e)$. Then $W(f) \subseteq W(e)$ and $D(e) \subseteq D(f)$. Let $x e y, x^{\prime} e y^{\prime}, s f t$ be in standard form such that

$$
p_{e, f}(x e y)=p_{e, f}\left(x^{\prime} e y^{\prime}\right)=s f t
$$

Let $z_{e}=v y_{1}$, where $v \in W(f) \cap W_{*}(e)$ and $y_{1} \in D(f)^{-1} \cap W_{*}(e)$. Let $u \in W(f) \subseteq W(e)$. Then

$$
\begin{aligned}
u\left(y_{1} y\right) & =\left(u y_{1}\right) y \\
& =\left(u * y_{1}\right) y \quad \text { since } \quad y_{1} \in D(f)^{-1} \\
& =\left(u * y_{1}\right) * y, \quad \text { since } \quad u y_{1} \in W(e), y \in D(e)^{-1} \\
& =u *\left(y_{1} y\right)
\end{aligned}
$$

So $y_{1} y \in D(f)^{-1}$. Similarly $y_{1} y^{\prime} \in D(f)^{-1}$. By (25),

$$
y_{1} y=t=y_{1} y^{\prime}, \quad x \Delta v=s=x^{\prime} \triangle v
$$

So $y=y^{\prime}$. Since $v \in W_{*}(e), x=x \Delta v$ and $x^{\prime}=x^{\prime} \Delta v$. So $x=x^{\prime}$. Thus $p_{e, f}$ is $1-1$. 
Assume conversely that $p_{e, f}$ is $1-1$. Let $v_{e}, v_{f}$ denote the longest elements of $W(e)$ and $W(f)$ respectively. Thus $v_{e} w_{0}$ and $v_{f} w_{0}$ are respectively the longest elements of $D(e)^{-1}$ and $D(f)^{-1}$. Let

$$
z_{e} v_{e} w_{0}=v y, v \in W(f), y \in D(f)^{-1}
$$

Then

$$
p_{e, f}\left(e v_{e} w_{0}\right)=f y=p_{e, f}\left(v^{-1} e v_{e} w_{0}\right)
$$

Since $p_{e, f}$ is $1-1, e v_{e} w_{0}=v^{-1} e v_{e} w_{0}$. So $v \in W_{*}(e)$. So $z=v^{-1} z_{e} \in W_{*}(e)$ and by (26),

$$
z v_{e} w_{0}=z *\left(v_{e} w_{0}\right)=y \leq v_{f} w_{0}
$$

So $v_{e} w_{0} \leq v_{f} w_{0}$. Hence $v_{f} \leq v_{e}$. Thus $W(f) \subseteq W(e)$ and $\lambda(f) \subseteq \lambda(e)$. This completes the proof.

Example 2.2 Let $G=G L_{3}(k), M=M_{3}(k)$. Then $\mathrm{W}$ is the group of permutation matrices and $\mathrm{R}$ is the monoid of partial permutation matrices (rook monoid). Let

$$
\begin{aligned}
& e=\left[\begin{array}{lll}
1 & 0 & 0 \\
0 & 0 & 0 \\
0 & 0 & 0
\end{array}\right], \\
& f=\left[\begin{array}{lll}
1 & 0 & 0 \\
0 & 1 & 0 \\
0 & 0 & 0
\end{array}\right] .
\end{aligned}
$$

Then $\lambda(e)=\lambda_{*}(e)=\{(23)\}, \lambda(f)=\{(12)\}, \lambda_{*}(f)=\emptyset$. Hence $p_{e, f}$ is not $1-1$ or onto. $p_{e, f}$ is given in Table 1. Since $\lambda_{*}(I)=\theta, p_{f, I}$ is onto. $p_{f, I}$ is given in Table 2. Combining with [7; figures 3 and 4], one obtains the Hasse diagram of $R$.

Example 2.3 Let $\phi: M_{n}(k) \rightarrow M_{N}(k)$ be defined as:

$$
\phi(A)=A \otimes \wedge^{2} A \otimes \cdots \otimes \wedge^{n} A
$$

where

$$
N=\prod_{r=1}^{n}\left(\begin{array}{l}
n \\
r
\end{array}\right)
$$


Table 1. Projection from rank 1 to rank 2.

\begin{tabular}{lll}
\hline$\sigma$ & $p_{e, f}(\sigma)$ \\
\hline$\left[\begin{array}{lll}1 & 0 & 0 \\
0 & 0 & 0 \\
0 & 0 & 0\end{array}\right]$ & {$\left[\begin{array}{lll}1 & 0 & 0 \\
0 & 0 & 1 \\
0 & 0 & 0\end{array}\right]$}
\end{tabular}

$\left[\begin{array}{lll}0 & 1 & 0 \\ 0 & 0 & 0 \\ 0 & 0 & 0\end{array}\right] \quad\left[\begin{array}{lll}0 & 1 & 0 \\ 0 & 0 & 1 \\ 0 & 0 & 0\end{array}\right]$

$\left[\begin{array}{lll}0 & 0 & 1 \\ 0 & 0 & 0 \\ 0 & 0 & 0\end{array}\right] \quad\left[\begin{array}{lll}0 & 1 & 0 \\ 0 & 0 & 1 \\ 0 & 0 & 0\end{array}\right]$

$\left[\begin{array}{lll}0 & 0 & 0 \\ 1 & 0 & 0 \\ 0 & 0 & 0\end{array}\right] \quad\left[\begin{array}{lll}0 & 0 & 1 \\ 1 & 0 & 0 \\ 0 & 0 & 0\end{array}\right]$

$\left[\begin{array}{lll}0 & 0 & 0 \\ 0 & 1 & 0 \\ 0 & 0 & 0\end{array}\right] \quad\left[\begin{array}{lll}0 & 0 & 1 \\ 0 & 1 & 0 \\ 0 & 0 & 0\end{array}\right]$

$\left[\begin{array}{lll}0 & 0 & 0 \\ 0 & 0 & 1 \\ 0 & 0 & 0\end{array}\right] \quad\left[\begin{array}{lll}0 & 1 & 0 \\ 0 & 0 & 1 \\ 0 & 0 & 0\end{array}\right]$

$\left[\begin{array}{lll}0 & 0 & 0 \\ 0 & 0 & 0 \\ 1 & 0 & 0\end{array}\right] \quad\left[\begin{array}{lll}0 & 0 & 1 \\ 0 & 0 & 0 \\ 1 & 0 & 0\end{array}\right]$

$\left[\begin{array}{lll}0 & 0 & 0 \\ 0 & 0 & 0 \\ 0 & 1 & 0\end{array}\right] \quad\left[\begin{array}{lll}0 & 0 & 1 \\ 0 & 0 & 0 \\ 0 & 1 & 0\end{array}\right]$

$\left[\begin{array}{lll}0 & 0 & 0 \\ 0 & 0 & 0 \\ 0 & 0 & 1\end{array}\right] \quad\left[\begin{array}{lll}0 & 1 & 0 \\ 0 & 0 & 0 \\ 0 & 0 & 1\end{array}\right]$

Let $M$ denote the Zariski closure of $\phi\left(M_{n}(k)\right)$ in $M_{N}(k)$. Then $W$ is the symmetric group of degree $n$ and $S=\{(12),(23), \ldots,(n-1 \quad n)\}$. Also

$\Lambda=\left\{e_{I} \mid I \subseteq S\right\} \cup\{0\}$

with

$$
e_{K} \leq e_{I} \Leftrightarrow K \subseteq I
$$


Table 2. Projection from rank 2 to rank 3.

\begin{tabular}{|c|c|c|c|c|c|c|c|c|c|c|c|}
\hline \multirow{2}{*}{\multicolumn{3}{|c|}{$\frac{\sigma}{\left[\begin{array}{lll}0 & 0 & 0\end{array}\right]}$}} & \multicolumn{3}{|c|}{$p_{f, I}(\sigma)$} & \multicolumn{3}{|c|}{$\sigma$} & \multicolumn{3}{|c|}{$p_{f, I}(\sigma)$} \\
\hline & & & & & & {$[0$} & 0 & & & & \\
\hline & 10 & & & 1 & & 0 & 1 & & 0 & 1 & \\
\hline & 00 & & & 0 & 0 & L & 0 & 1 & Lo & 0 & 1 \\
\hline & 0 & 07 & & 1 & 07 & {$[0$} & 0 & 1 & {$[1$} & 0 & 0 \\
\hline & 0 & & & 0 & & 0 & 0 & 0 & 0 & 0 & 1 \\
\hline $\mathrm{L}^{1}$ & 0 & 0 & $\lfloor 1$ & 0 & & {$\left[\begin{array}{ll}0 \\
{[}\end{array}\right.$} & 1 & 0 & LO & 1 & 0 \\
\hline$[0$ & 0 & 07 & & 0 & 17 & {$[0$} & 0 & 1 & {$[0$} & 1 & 0 \\
\hline & 0 & & & 0 & & 1 & 0 & 0 & 1 & 0 & 0 \\
\hline-0 & 1 & 0 & 0 & 1 & 0 & 0 & 0 & 0 & {$[0$} & 0 & 1 \\
\hline$[0$ & 1 & $0]$ & & 1 & & {$[1$} & 0 & 0 & $\Gamma 1$ & 0 & 0 \\
\hline 0 & 0 & 0 & & 0 & & 0 & 0 & 0 & 0 & 1 & 0 \\
\hline 1 & 0 & $0]$ & L1 & 0 & $0\rfloor$ & 0 & 0 & 1 & LO & 0 & 1 \\
\hline$[0$ & 0 & 07 & & 0 & 07 & {$[1$} & 0 & 0 & {$[1$} & 0 & 0 \\
\hline & 0 & & & 0 & & 0 & 1 & 0 & 0 & 1 & 0 \\
\hline 0 & 1 & $0\rfloor$ & 0 & 1 & 0 & {$\left[\begin{array}{ll}0 \\
0\end{array}\right.$} & 0 & 0 & {$[0$} & 0 & 1 \\
\hline$[0$ & 1 & 07 & & 1 & $0]$ & {$[0$} & 0 & 1 & {$[1$} & 0 & 0 \\
\hline 1 & 0 & 0 & & 0 & & 0 & 1 & 0 & 0 & 1 & 0 \\
\hline-0 & 0 & 0 & Lo & 0 & $1]$ & 0 & 0 & 0 & Lo & 0 & 1 \\
\hline 0 & 0 & 07 & & 1 & $0]$ & {$[0$} & 1 & 0 & $\lceil 1$ & 0 & 0 \\
\hline & 0 & 0 & & 0 & & 0 & 0 & 0 & 0 & 1 & 0 \\
\hline L & 0 & $1\rfloor$ & L & 0 & $1]$ & Lo & 0 & 1 & Lo & 0 & 1 \\
\hline$\Gamma 0$ & 0 & 17 & & 1 & $0]$ & {$[1$} & 0 & 0 & {$[1$} & 0 & 0 \\
\hline 0 & 0 & 0 & & 0 & & 0 & 0 & 1 & 0 & 1 & 0 \\
\hline L & 0 & $0\rfloor$ & L1 & 0 & 0 & {$\left[\begin{array}{ll}0 \\
0\end{array}\right.$} & 0 & 0 & Lo & 0 & 1 \\
\hline$\Gamma 1$ & 0 & 07 & & 0 & $0]$ & {$[0$} & 1 & 0 & {$[1$} & 0 & 0 \\
\hline 0 & 0 & 0 & & 0 & & c & 0 & 1 & 0 & 1 & 0 \\
\hline$\left[\begin{array}{lll}0 & \\
0\end{array}\right.$ & 1 & $0\rfloor$ & 0 & 1 & $0\rfloor$ & L & 0 & 0 & Lo & 0 & 1 \\
\hline
\end{tabular}

and

$$
\lambda\left(e_{I}\right)=\lambda^{*}\left(e_{I}\right)=I, \quad \lambda_{*}\left(e_{I}\right)=\emptyset, \quad I \subseteq S
$$

So by Theorem 2.1, $p_{e_{K}, e_{I}}$ is onto for $K \subseteq I$.

Example 2.4 Let $\phi: S L_{n}(k) \rightarrow G L_{N}(k)$ be defined as:

$$
\phi(A)=A \oplus \wedge^{2} A \oplus \cdots \oplus \wedge^{n} A
$$


where $N=2^{n}-1$. Let $M$ denote Zariski closure in $M_{N}(k)$ of $k \phi\left(S L_{n}(k)\right)$. Again $W$ is symmetric group of degree $n$ and $S=\{(12),(23),(n-1 \quad n)\}$. Then

$$
\Lambda=\{1\} \cup\left\{e_{I} \mid I \subseteq S\right\}
$$

with

$$
e_{I} \leq e_{K} \Leftrightarrow K \subseteq I
$$

and

$$
\lambda\left(e_{I}\right)=\lambda_{*}\left(e_{I}\right)=I, \quad \lambda^{*}\left(e_{I}\right)=\emptyset, \quad I \subseteq S
$$

So by Theorem 2.1, $p_{e_{I}, e_{K}}$ is $1-1$ for $K \subseteq I$.

Corollary 2.5 Lete $<f$ in $\Lambda, \sigma \in W e W, \theta \in W f W$. Let $\sigma=x e y$ in standardform, $z_{f} z_{e} y=$ $u y_{1}$ with $u \in W(f), y_{1} \in D(f)^{-1}$. Then $\theta$ covers $\sigma$ if and only if $f$ covers $e$ in $\Lambda, p_{e, f}(\sigma)=\theta$ and $l(x u)=l(x)-l(u)$.

Proof: If $\theta$ covers $\sigma$, then by Theorem 2.1, $f$ covers $e$ in $\Lambda$ and $\theta=p_{e, f}(\sigma)$. So assume that $f$ covers $e$ in $\Lambda$ and $\theta=p_{e, f}(\sigma)$. The maximum elements of $W e W$ and $W f W$ are respectively $w_{0} z_{e} e$ and $w_{0} z_{f} f$. Since $f$ covers $e$, we see by (9) and [6; Chapter 10] that

$$
\lambda_{*}(f)=\lambda(f) \cap \lambda_{*}(e)
$$

So by (22),

$$
p_{e, f}\left(w_{0} z_{e} e\right)=w_{0} z_{e} f z_{f} z_{e}
$$

covers $w_{0} z_{e} e$. By (19), (20), [ $\left.\sigma, w_{0} z_{e}\right]$ has length

$$
l\left(w_{0} z_{e}\right)-l(x)+l(y)
$$

and $\left[w_{0} f z_{f} z_{e}, w_{0} z_{f} f\right]$ has length

$$
l\left(w_{0} z_{f}\right)-l\left(w_{0} z_{e}\right)+l\left(z_{f} z_{e}\right)
$$

By (27)-(29), $\left[\sigma, w_{0} z_{f} f\right]$ has length

$$
l\left(w_{0} z_{f}\right)-l(x)+l(y)+l\left(z_{f} z_{e}\right)+1
$$

By (22), $\theta=(x \Delta u) f y_{1}$. Also

$$
l(u)+l\left(y_{1}\right)=l\left(z_{f} z_{e} y\right)=l\left(z_{f} z_{e}\right)+l(y)
$$


By (19), (20), $\left[\theta, w_{0} z_{f} f\right]$ has length

$$
l\left(w_{0} z_{f}\right)-l(x \Delta u)+l\left(y_{1}\right)
$$

By (30)-(32), $[\sigma, \theta]$ has length

$$
l(x \Delta u)+l(u)-l(x)+1
$$

Hence $\theta$ covers $\sigma$ if and only if

$$
l(x \Delta u)=l(x)-l(u)
$$

By Lemma 1.1, this is true if and only if $l(x \Delta u)=l(x u)$. This completes the proof.

Corollary 2.6 Any interval in $R$ of length 2 has at most 4 elements.

Proof: Consider an interval $[\sigma, \theta]$ in $R$ of length 2. Let $\sigma \in W e W, \theta=W f W$. Then $e \leq f$.

Case 1. $e=f$. By (14), WeW is isomorphic to the dual of $\mathcal{W}_{I, K}^{*}$ where $I=\lambda(e)$ and $K=\lambda_{*}(e)$. Now $\mathcal{W}_{I, K}^{*}$ is a subposet of $\mathcal{W}_{I, \emptyset}^{*}$ with the same rank function. By [7; Theorem 3.3], $\mathcal{W}_{I, \emptyset}^{*}$ is an Eulerian poset. Hence any interval of length 2 in $\mathcal{W}_{I, \emptyset}^{*}$ has 4 elements. It follows that $|[\sigma, \theta]| \leq 4$ in $W e W$.

Case 2. $e<f$ and $f$ does not cover $e$ in $\Lambda$. Then by Corollary 2.5, $[e, f]$ has length 2 in $\Lambda$. Now $E(\bar{T})$ is the face lattice of a polytope. Hence in $\Lambda,|[e, f]| \leq 4$. So in $\Lambda$,

$$
[e, f]=\left\{e, h, h^{\prime}, f\right\}
$$

with $e<h, e<h^{\prime}<f$ and with the possibility that $h=h^{\prime}$. So by Theorem 2.1,

$$
[\sigma, \theta]=\left\{\sigma, p_{e, h}(\sigma), p_{e, h^{\prime}}(\sigma), \theta\right\}
$$

in $R$.

Case 3. $f$ covers $e$ in $\Lambda$ and $\theta=p_{e, f}(\sigma)$. Let $\sigma=x e y$ in standard form. If $\pi \in(\sigma, \theta)$, then $\pi \in W e W$ and $\pi$ covers $\sigma$. So by (14), either $R \pi=R \sigma$ or $\pi R=\sigma R$. Let $\pi_{1}, \pi_{2} \in(\sigma, \theta)$ such that $R \pi_{1}=R \sigma=R \pi_{2}$. Then $\pi_{1}=x_{1} e y, \pi_{2}=x_{2} e y$ in standard form. Let $z_{f} z_{e} y=$ $u y_{1}, u \in W(f), y_{1} \in D(f)^{-1}$. Since $\theta$ covers $\pi_{1}$ and $\pi_{2}$, we see by Corollary 2.5 that

$$
x_{1} u f y_{1}=p_{e, f}\left(\pi_{1}\right)=\theta=p_{e, f}\left(\pi_{2}\right)=x_{2} u f y_{1}
$$

in standard form. It follows that $x_{1} u=x_{2} u$. Hence $x_{1}=x_{2}$ and $\pi_{1}=\pi_{2}$. Dually by (7), $\pi_{1} R=\pi_{2} R$ implies that $\pi_{1}=\pi_{2}$. It follows that $|[\sigma, \theta]| \leq 4$. 
Case 4. $f$ covers $e$ in $\Lambda$ and $p_{e, f}(\sigma)=\theta_{1}<\theta$. Then $\theta_{1}$ covers $\sigma$ and $\theta$ covers $\theta_{1}$. Let $\pi_{1}, \pi_{2} \in(\sigma, \theta), \pi_{1} \neq \pi_{2}, \pi_{1} \neq \theta_{1}, \pi_{2} \neq \theta_{1}$. Then $\pi_{1}, \pi_{2} \in W e W$ and $\theta$ covers $\pi_{1}, \pi_{2}$. So $p_{e, f}\left(\pi_{1}\right)=\theta=p_{e, f}\left(\pi_{2}\right)$. Since $\pi_{1}, \pi_{2} \operatorname{cover} \sigma$, we see by (14) that for $i=1,2, \pi_{i} R=\sigma R$, or $R \sigma=R \pi_{i}$. Since $\pi_{1} \neq \pi_{2}$, we can assume by (33) that $R \pi_{1}=R \sigma, R \pi_{2} \neq R \sigma$. So $\pi_{1}=$ $x^{\prime} e y, \pi_{2}=x e y^{\prime}$ in standard form, $x^{\prime}$ covers $x$ and $y$ covers $y^{\prime}$. Since $\theta$ covers $\pi_{1}, \pi_{2}$,

$$
z_{f} z_{e} y=u y_{1}, z_{f} z_{e} y^{\prime}=v y_{1}, u, v \in W(f), y_{1} \in D(f)^{-1}
$$

Then $\theta_{1}=x_{1} f y_{1}, \theta=x_{1}^{\prime} f y_{1}$ in standard form with

$$
x_{1}=x u, \quad x_{1}^{\prime}=x v=x^{\prime} u
$$

and by Corollary 2.5 ,

$$
x=x_{1} * u^{-1}=x_{1}^{\prime} * v^{-1}, \quad x^{\prime}=x_{1}^{\prime} * u^{-1}
$$

Now $x_{1}^{\prime}$ covers $x_{1}$ and hence $u^{-1}$ covers $v^{-1}$ by Corollary 1.2. Since $x^{\prime}$ covers $x$, this contradicts the exchange condition for $W$. So $|[\sigma, \theta]| \leq 4$, completing the proof.

Corollary 2.6 leads us to the following conjecture concerning the Möbius function $\mu$ on $R$. We refer to [17; Chapter 3] for the theory of Möbius functions on posets:

Conjecture 2.7 Let $\sigma, \theta \in R, \sigma \leq \theta$. Then

$$
\mu(\sigma, \theta)= \begin{cases}(-1)^{l[\sigma, \theta]} & \text { if every interval of length } 2 \text { in }[\sigma, \theta] \text { has } 4 \text { elements } \\ 0 & \text { otherwise }\end{cases}
$$

Here $l[\sigma, \theta]$ denotes the length of the interval $[\sigma, \theta]$.

Theorem 3.4 below establishes Conjecture 2.7 for canonical monoids.

\section{Canonical monoids}

In this section we will assume that $M$ is a canonical monoid. This means that $\Lambda^{*}=\Lambda \backslash\{0\}$ has a least element $e_{0}$ with $\lambda\left(e_{0}\right)=\emptyset$. Then as in Example 2.3, $\Lambda^{*}$ is in $1-1$ correspondence with the subsets of $S$. So we can write:

$$
\Lambda^{*}=\left\{e_{I} \mid I \subseteq S\right\}
$$

with

$$
\lambda\left(e_{I}\right)=\lambda^{*}\left(e_{I}\right)=I, \lambda_{*}\left(e_{I}\right)=\emptyset, I \subseteq S
$$


and

$$
e_{K} \leq e_{I} \Leftrightarrow K \subseteq I
$$

See $[9,13]$ for details. Example 2.3 is an example of a canonical monoid. More generally if $G_{0}$ is a semisimple group and if $\phi: G_{0} \rightarrow G L_{n}(k)$ is an irreducible representation with highest weight in the interior of the Weyl chamber, then the Zariski closure in $M_{n}(k)$ of $k \phi\left(G_{0}\right)$ is a canonical monoid. Canonical monoids are closely related to canonical compactifications of semisimple groups in the sense of [2]. The connection between reductive monoids and embeddings of homogenous spaces is studied in [12]. See also [19]. Basically the canonical compactification is obtained as the projective variety $X=(M \backslash\{0\}) /$ center. Then the $B \times B$-orbits of $X$ are indexed by $R^{*}=R \backslash\{0\}$. See [13]. The Bruhat-Chevalley order on $R^{*}$ corresponds to the Zariski closure inclusion of $B \times B$-orbits of $X$, the geometric properties of which have been studied in [15].

Let $M$ be a canonical monoid. For $I \subseteq S$, let $R_{I}=W e_{I} W=W e_{I} D_{I}^{-1}$. Then by (5), (34),

$$
R^{*}=R \backslash\{0\}=\bigsqcup_{I \subseteq S} R_{I}
$$

For $K \subseteq I$, we write $p_{K, I}$ for $p_{e_{K}, e_{I}}$. So $p_{K, I}: R_{K} \rightarrow R_{I}$. By [7; Theorem 3.3], each $R_{I}$ is an Eulerian poset. We will show in this section that $R^{*}$ is an Eulerian poset.

Lemma 3.1 Let $\sigma \in R_{\emptyset}, s \in W$ such that $p_{\emptyset, S}(\sigma)<s$. Then $[\sigma, s] \cap R_{\emptyset}$ is balanced.

Proof: Let $e=e_{\emptyset}, \sigma=x_{0} e y_{0}, s_{0}=p_{\emptyset, S}(\sigma)=x_{0} \Delta y_{0}$. Then $s_{0}<s$. For $y \leq y_{0}$, let

$$
A_{y}=[\sigma, s] \cap \text { Wey }=\left\{\text { xey } \mid x_{0} \leq x \leq s \circ y^{-1}\right\}
$$

Thus $A_{y}$ is a non-trivial interval in $R_{\emptyset}$ unless $x_{0}=s \circ y^{-1}$. So $A_{y}$ is balanced unless $x_{0}=s \circ y^{-1}$. By Lemma 1.3,

$$
Y=\left\{y \leq y_{0} \mid x_{0}=s \circ y^{-1}\right\}
$$

is balanced. It follows that $[\sigma, s] \cap R_{0}$ is balanced.

Corollary 3.2 Let $\sigma \in R_{\emptyset}, \theta \in R_{I}$ such that $p_{\emptyset, I}(\sigma)<\theta$. Then $[\sigma, \theta] \cap R_{\emptyset}$ is balanced.

Proof: Let $e=e_{\emptyset}, f=e_{I}, \sigma=x_{0} e y_{0}, \theta=s f t$ in standard form. Suppose $x_{0} \notin s W_{I}$. For $t \leq y \leq y_{0}$, let

$$
A_{y}=[\sigma, \theta] \cap W e y
$$

By [3],

$$
w=w_{y}=\max \left\{u \in W_{I} \mid u^{-1} t \leq y\right\}
$$


exists. Let $x e y=A_{y}$. Then $x_{0} e y_{0} \leq x e y \leq s f t$. So $x_{0} \leq x$ and there exists $u \in W_{I}$ such that $x \leq s u, u^{-1} t \leq y$. Then $u \leq w$ and $s u \leq s \circ u \leq s \circ w$. Also $s \circ w=s w_{1}$ for some $w_{1} \leq w$ and $w_{1}^{-1} t \leq w^{-1} t \leq y$. Hence

$$
\sigma \leq x_{0} e y \leq x e y \leq(s \circ w) e y \leq \theta
$$

So

$$
A_{y}=\left[x_{0} e y,(s \circ w) e y\right]
$$

Since $x_{0} \notin s W_{I}, x_{0} \neq s \circ w$. Since $R_{\emptyset}$ is Eulerian, $A_{y}$ is balanced. Thus

$$
[\sigma, \theta] \cap R_{\emptyset}=\bigcup_{t \leq y \leq y_{0}} A_{y}
$$

is balanced. Similarly if $y_{0} \notin W_{I} t,[\sigma, \theta] \cap R_{\emptyset}$ is balanced. So let $x_{0} \in s W_{I}, y_{0} \in W_{I} t$. So $s=s_{1} v, x_{0}=s_{1} x_{1}, y_{0}=y_{1} t$ for some $s_{1} \in D_{1}, v, x_{1}, y_{1} \in W_{I}$. If $\sigma^{\prime}=x_{1} e y_{1}, \theta^{\prime}=v e_{I}$, then working in $e_{I} R e_{I}$, we see by Lemma 3.1 that $\left[\sigma^{\prime}, \theta^{\prime}\right] \cap R_{\emptyset}$ is balanced. Hence

$$
[\sigma, \theta] \cap R_{\emptyset}=s_{1}\left(\left[\sigma^{\prime}, \theta^{\prime}\right] \cap R_{\emptyset}\right) t
$$

is balanced.

Lemma 3.3 Let $\sigma \in R_{\emptyset}, \theta \in R_{I}, \sigma \leq \theta$. Let

$$
Z=\left\{\pi \in[\sigma, \theta] \cap R_{\emptyset} \mid p_{\emptyset, I}(\pi)=\theta\right\}
$$

Then

$$
(-1)^{l\left(e_{\emptyset}\right)} \sum_{\pi \in Z}(-1)^{l(\pi)}=(-1)^{l\left(e_{I}\right)}(-1)^{l(\theta)}
$$

Proof: We prove by induction on $l(\theta)$. Suppose first that $p_{\emptyset, I}(\sigma)=\theta^{\prime}<\theta$. For $\delta \in\left[\theta^{\prime}, \theta\right]$ let

$$
Z_{\delta}=\left\{\pi \in[\sigma, \theta] \cap R_{\emptyset} \mid p_{\emptyset, I}(\sigma)=\delta\right\}
$$

Then $Z=Z_{\theta}$ and

$$
[\sigma, \theta] \cap R_{\emptyset}=\bigsqcup_{\delta \in\left[\theta^{\prime}, \theta\right]} Z_{\delta}
$$


PUTCHA

By induction hypothesis,

$$
(-1)^{l\left(e_{\emptyset}\right)} \sum_{\pi \in Z_{\delta}}(-1)^{l(\pi)}=(-1)^{l\left(e_{I}\right)}(-1)^{l(\delta)}
$$

for $\delta \in\left[\theta^{\prime}, \theta\right)$. By Corollary 3.2,

$$
\sum_{\pi \in[\sigma, \theta] \cap R_{\emptyset}}(-1)^{l(\pi)}=0=\sum_{\delta \in\left[\theta^{\prime}, \theta\right]}(-1)^{l(\delta)}
$$

By (36)-(38) we see that (37) is also valid for $\delta=\theta$.

Assume therefore that $p_{\emptyset, I}(\sigma)=\theta$. Then as in the proof of Corollary 3.2, we may assume that $\theta=s \in W$. Let $e=e_{\emptyset}, \sigma=x_{0} e y_{0}$. Then $x_{0} \triangle y_{0}=s$. So $x_{0} y_{1}=s, s * y^{-1}=x_{0}$ with $y_{1} \leq y_{0}$. For $y \leq y_{0}$, let

$$
A_{y}=[\sigma, \theta] \cap W e y
$$

Let $x e y \in A_{y}$. Then $x_{0} e y_{0} \leq x e y \leq s$. So $x \leq s \circ y^{-1}$. Hence

$$
\sigma \leq x_{0} e y \leq x e y \leq\left(s \circ y^{-1}\right) e y \leq s
$$

So

$$
A_{y}=\left[x_{0} e y,\left(s \circ y^{-1}\right) e y\right]
$$

and

$$
Z=[\sigma, s] \cap R_{\emptyset}=\bigsqcup_{y \leq y_{0}} A_{y}
$$

If $A_{y} \neq \emptyset$, then since $p_{\emptyset, S}(\sigma)=s$, we see that $x_{0} \Delta y=s$. So if $x_{0}=s \circ y^{-1}$, then by Lemma 1.1 (vi), $x_{0}=s * y^{-1}$. Hence $y=y_{1}$. Moreover $A_{y_{1}}=\left\{x_{0} e y_{1}\right\}$ and by (19), $l\left(x_{0} e y_{1}\right)=l(e)+l(s)$. If $A_{y} \neq \emptyset$ and $x_{0} \neq s \circ y^{-1}$, then $A_{y}$ is balanced by (40). The result follows.

Theorem 3.4 $R^{*}$ is an Eulerian poset.

Proof: Let $\sigma, \theta \in S^{*}, \sigma<\theta$. We need to show that $[\sigma, \theta]$ is balanced. Let $\sigma \in R_{K}, \theta \in$ $R_{I}$. So $K \subseteq I$. First assume that $p_{K, I}(\sigma)<\theta$. By Theorem 2.1 (vi), there exists $\sigma_{0} \in R_{\emptyset}$ such that $p_{\emptyset, K}\left(\sigma_{0}\right)=\sigma$. By Corollary 3.2, $\left[\sigma_{0}, \theta\right] \cap R_{\emptyset}$ is balanced. So by Lemma 3.3, $\left[\sigma_{0}, \theta\right] \cap R_{L}$ is balanced for $L \subseteq S$. For $I \subseteq J \subseteq S,\left[\sigma_{0}, \theta\right] \cap R_{J}=[\sigma, \theta] \cap R_{J}$ by Thoerem 2.1. It follows that $[\sigma, \theta]$ is balanced.

Now assume that $p_{K, I}(\sigma)=\theta$. Let $\theta=s \theta^{\prime} t, s \in D_{I}, t \in D_{I}^{-1}, \theta^{\prime} \in W_{I} e_{I}$. By (21), $\sigma^{\prime}=s^{-1} \sigma t^{-1} \in W_{I} e_{K} W_{I}$. Then $[\sigma, \theta]=s\left[\sigma^{\prime}, \theta^{\prime}\right] t \cong\left[\sigma^{\prime}, \theta^{\prime}\right]$. Thus without loss of 
generality, we may assume that $\theta=s \in W$. Let $\sigma=x_{0} e_{K} y_{0}$ in standard form, $K \neq S$. Then by (21), $s=x_{0} \triangle y_{0}$. Hence $x_{0}=s * y_{1}^{-1}$ for some $y_{1} \leq y_{0}$. By Corollary 2.5,

$$
\Omega=\left\{x_{0} e_{J} y_{1} \mid K \subseteq J \subseteq S\right\}=\left[x_{0} e_{K} y_{1}, s\right]
$$

Let

$$
y_{1}=u_{J} v_{J}, \quad u_{j} \in W(J), \quad v_{J} \in D_{J}^{-1}, \quad K \subseteq J \subseteq S
$$

For $y \in D_{J}^{-1}$, let

$$
A_{J}(y)=[\sigma, s] \cap W e_{J} y
$$

Suppose $y \in D_{J}^{-1}, y \neq v_{J}$ and $x e_{J} y \in A_{J}(y)$. Then

$$
\sigma \leq x e_{J} y \leq s
$$

Then $y \leq y_{0}$ and by [3],

$$
w=\max \left\{u \in W_{J} \mid u y \leq y_{0}\right\}
$$

exists. By (16), (43), there exists $u \in W_{J}$ such that $x_{0} \leq x * u^{-1}, u y \leq y_{0}$. So $u \leq w$. By Lemma 1.1,

$$
x_{0} \Delta w \leq x_{0} \Delta u \leq\left(x * u^{-1}\right) \Delta u=x
$$

Hence

$$
\sigma \leq\left(x_{0} \Delta w\right) e_{J} y \leq x e_{J} y
$$

Also by (16), (43),

$$
\sigma \leq x e_{J} y \leq s \circ y^{-1} e_{J} y \leq s
$$

Since $p_{K, S}(\sigma)=s$, we see that $x \Delta y=s$. So by Lemma 1.1 (vi), $s \circ y^{-1}=s * y^{-1}$. Thus by (44), (45),

$$
A_{J}(y)=\left[\left(x_{0} \Delta w\right) e_{J} y,\left(s * y^{-1}\right) e_{J} y\right]
$$

Suppose $\left|A_{J}(y)\right|=1$. Then $x_{0} \Delta w=s * y^{-1}$. By Lemma 1.1, $\left(x_{0} \Delta w\right) * w_{1}^{-1}=x_{0}$ for some $w_{1} \leq w$. Then

$$
s * y_{1}^{-1}=x_{0}=\left(x_{0} \triangle w\right) * w_{1}^{-1}=s * y^{-1} * w_{1}^{-1}
$$


By Corollary 1.2, $y_{1}^{-1}=y^{-1} * w_{1}^{-1}$. So $y=w_{1} y$. Since $w_{1} \in W(J)$ and $y \in D_{J}^{-1}$, we see by (42) that $y=v_{J}$, a contradiction. Hence $A_{J}(y)$ is a non-trivial interval in $R_{J}$ and hence balanced.

Assume next that $y=v_{J}$. Let $x e_{J} v_{J} \in R_{J}$. Then

$$
\sigma=x_{0} e_{K} y_{0} \leq x e_{J} v_{J} \leq s
$$

Since $p_{J, S}\left(x e_{J} y\right)=s$, we see by Theorem 2.1 that $x \Delta v_{J}=s$. By Lemma 1.1,

$$
x=s * v^{-1} \quad \text { for some } \quad v \leq v_{J}
$$

Also by (16), $x_{0} \leq x * u$ for some $u \in W(J)$. Hence by (47),

$$
s * y_{1}^{-1}=x_{0} \leq x * u=s * v^{-1} * u
$$

By Corollary 1.2, $y_{1}^{-1} \leq v^{-1} u$. So $y_{1} \leq u^{-1} v$. So we see by (42), (47) that $v=v_{J}$. So $x=s v_{J}^{-1}$. Hence

$$
x e_{J} y=s v_{J}^{-1} e_{J} v_{J}=s v_{J}^{-1} u_{J}^{-1} e_{J} u_{J} v_{J}=s y_{1}^{-1} e_{J} y_{1}=x_{0} e_{J} y_{1}
$$

Thus $A_{J}\left(v_{J}\right)=\left\{x_{0} e_{J} y_{1}\right\}$. It follows that $[\sigma, s] \backslash \Omega$ is balanced. By (41), $\Omega \cong 2^{S \backslash K}$ is also balanced. Hence $[\sigma, s]$ is balanced, completing the proof.

Example 3.5 $M_{2}(k)$ is a canonical monoid. The Eulerian poset $R^{*}$ is given by:

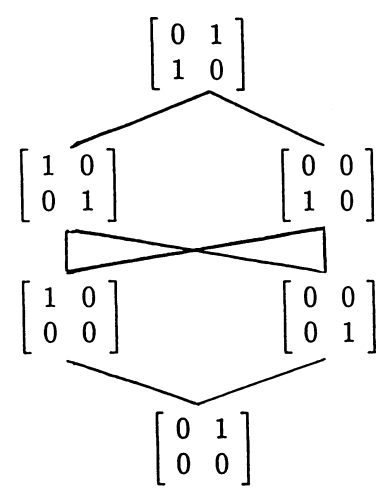

$M_{3}(k)$ is not a canonical monoid. In this case, Example 2.2 shows that $R^{*}$ is not Eulerian. The monoids in Example 2.3 are canonical. With $n=3, R^{*}$ will be an Eulerian poset with 78 elements. 


\section{References}

1. C. Chevalley, "Sur les décompositions cellulaires des espaces $G / B$," in Algebraic groups and their generalizations, Proc. Symp. Pure Math 56 Amer. Math. Soc. (1991), 1-23.

2. C. DeConcini, "Equivariant embeddings of homogenous spaces," Proc. Internat. Congress of Mathematicians (1986), 369-377.

3. V.V. Deodhar, "A splitting criterion for the Bruhat orderings on Coxeter groups," Comm. Algebra 15 (1987), 1889-1894.

4. E.A. Pennell, M.S. Putcha, and L.E. Renner, "Analogue of the Bruhat-Chevalley order for reductive monoids," J. Algebra 196 (1997), 339-368.

5. M.S. Putcha, “A semigroup approach to linear algebraic groups," J. Algebra 80 (1983), 164-185.

6. M.S. Putcha, Linear Algebraic Monoids, London Math. Soc. Lecture Note Series 133, Cambridge Univ. Press, 1988.

7. M.S. Putcha, "Shellability in reductive monoids," Trans. Amer. Math. Soc. 354 (2001), 413-426.

8. M.S. Putcha and L.E. Renner, "The system of idempotents and the lattice of $\mathcal{J}$-classes of reductive algebraic monoids," J. Algebra 116 (1988), 385-399.

9. M.S. Putcha and L.E. Renner, "The canonical compactification of a finite group of Lie type," Trans. Amer. Math. Soc. 337 (1993), 305-319.

10. L.E. Renner, "Analogue of the Bruhat decomposition for reductive algebraic monoids," J. Algebra 101 (1986), 303-338.

11. L.E. Renner, "Analogue of the Bruhat decomposition for reductive algebraic monoids II. The length function and trichotomy," J. Algebra 175 (1995), 695-714.

12. L.E. Renner, "Classification of simisimple varieties," J. Algebra 122 (1989), 275-287.

13. L.E. Renner, "An explicit cell decomposition of the canonical compactification of an algebraic group," Can. Math. Bull 46 (2003), 140-148.

14. L. Solomon, "An introduction to reductive monoids," in Semigroups, Formal Languages and Groups (J. Fountain, ed.), Kluwer (1995) 293-352.

15. T.A. Springer, "Intersection cohomology of $B \times B$ orbit closures in group compactifications," J. Algebra 258 (2002), 71-111.

16. R.P. Stanley, "Some aspects of groups acting on finite posets," J. Comb. Theory A 32 (1982), 132-161.

17. R.P. Stanley, Enumerative Combinatorics vol. 1, Cambridge Studies in Advanced Math 49, Cambridge University Press, 1997.

18. D.-N. Verma, "Möbius inversion for the Bruhat ordering on a Weyl group," Ann. Sci. École Norm. Sup. 4 (1971), 393-398.

19. E.B. Vinberg, “ On reductive algebraic semigroups,” Amer. Math. Soc. Transl. Series 2169 (1994), $145-182$. 